\title{
IMPACT OF SALINITY SEED SPROUT CHARACTERIZATION OF FIVE FABA BEAN (Vicia faba L.) VARIETIES
}

\author{
Ali $^{*}$ M.A.A., Abdallah M.M.F., Nashwa A. Abo El-Azam \\ and Abou El-Yazeid A. \\ Horticulture Dept., Fac. of Agric., Ain Shams Univ., P.O. Box 68, Hadayek Shoubra, \\ 11241 Cairo, Egypt \\ *Corresponding author: mahmoud adel489@agr.asu.edu.eg
}

Received 25 July, 2019

Accepted 12 November, 2019

\section{ABSTRACT}

One of the unique properties of earlier stages of plant growth is germination. Germination is morphological expression of various metabolic activities in seeds. Plants exposure to the abiotic stresses for instance salinity that influence physiological processes, anatomy, developmental systems and plant development. Agriculture production under arid and semi-arid zone is low owning to many factors for example accumulation of salts in soils and water, total of land area under salinity is about 953 million ha. The aim of this research was to evaluate the germination characters of five faba bean (Vicia faba L.) varieties [Nubaria (1), Giza (843), Sakha (1), Sakha (3) and, Sakha (4)] were using saline solution containing $(0,1000$ and 2000 ppm $\mathrm{NaCl}$ ), were utilized to examine their water uptake, germination percentage, germination index, relative salt injury rate, radicle length, sprout fresh and dry weight, weight losses and seed volume using saline solution. Results indicated that the higher concentrations of salt have a negative impact on water uptake, germination percentage, radical length, sprout fresh and dry weight, seed volume, and relative salt injury rate. At all salinity concentrations, all varieties showed different degrees of salt tolerance. Sakha (3), (4) and Giza (843) had a better salt tolerance index than the others. Nubaria (1) and Sakha (1). Nubaria (1) Giza (843) had the highest percentage and index of germination, while Sakha (1) had the shorter radical length and susceptible to salt injury rate. Moreover, Giza (843) and Sakha (3) genotypes can be considered as tolerant to salt stress compared to the other ones. These genotypes also could be used in the breeding program for enhanc- ing faba bean cultivation in newly reclaimed lands. Data suggest a test of seed germination may be useful to use in developing new lines of faba bean to grow in saline soils.

Keywords: Faba bean seed germination, Water uptake, Relative salt injury rate, Salt tolerance index, Sprout character, Salinity stress

\section{INTRODUCTION}

Faba bean (Vicia faba L.) is a remarkable crop belonged to family Fabaceae, which contain crops such as soybean, chickpeas, peas, and beans. Faba bean is prevalent legume diet with significant yield capacity and high nutritional prominence for human and animals. Egypt is ranked seventh productions of faba been in the world after China, Ethiopia, Australia, United Kingdom, Germany, France (FAO, 2017). Faba bean is one of Important and unique legume crop planted in Egypt due to the protein, carbohydrates, dietary fiber and secondary metabolites such as phenolic and antioxidant value (Erba et al 2019; Hossain and Mortuza, 2006). An extensive problem in agriculture is salinity, as a major part of the abiotic stresses in arid and semi-arid zone and it affects the agriculture world land area (Kumawat et al 2017).

Seed germination is the decisive period in the life stages of plants. Germination processes include those steps that begin with the absorption of water by dry seed and end with the emergence of radical (Copeland and McDonald, 2001). Water imbibition with a seed is tri-phasic; phase 1 rapid initial imbibed; phase 2 is lull phase and then phase 3 an increment of water imbibition, but, only 
while germination happens (Ghazi, 1998; Manz, 2005 and Schopfer \& Plachy, 1984). Thus, salinity tolerance through sprouting is crucial for promotion of growing plants and under salt stress (Khan et al 2000). Despite, the sprouting importance for growing in saline soils, more is known of in stages of reproductive and vegetative about salt tolerance mechanism than during the sprouting stage (Ibrahim et al 2019 and Munns \& Tester, 2008). Since excess chloride and sodium ions imbalance has a deleterious effect at all stages of plant growth (Begum et al 2013; Zhang et al 2010), therefore tolerance metabolism to $\mathrm{Na}^{+}$and $\mathrm{Cl}^{-}$is important during sprouting than later stages of plant growth, owing to limited carbohydrates available in seed (Zhang et al 2010).

Salinity reduces the proficiency of plants to absorption water leading to a decrease in plant growth also metabolic changes (Kader and Jutzi, 2002). Experiment done on six wheat varieties under different salinity concentrations, indicated that water uptake decreased by increasing salinity concentration (Ibrahim et al 2016). Hence, salinity may reduce crop production by uncomfortable water and nutritional balance of the plant (Begum et al 2013; Santos et al 2018). Solution imbibe by root system restricted at high osmotic potential and toxicity effect due to an accumulation of sodium and chloride (Na-Cl) ions (Al-Karaki, 1997; Munns, 2002). Salt stress affects seed germination percentage, rate and seedling growth. Study on Sugar beet (Beta vulgaris), cabbage (Brassica oleracea capitata), amaranth (Amaranthus paniculatus) and pak-choi (Brassica compestris) seed using salt solution reducing to seed germination percentage rate and percentage also reducing seedling growth (root and shoot lengths and fresh root and shoot weights) (Jamil et al 2006). Moreover, three cultivars of pea (Pisum sativum L.) were investigate under $\mathrm{NaCl}$ solutions and the result was decreasing germination and seedling growth in all cultivars (Okçu et al 2005). In spinach experiment, salinity negatively reducing absorption efficiency, seed vigor, hypocotyl and root length (Ibrahim et al 2019). However, Kuntze (Limonium sinense), sieb (Glycine soja) and Stapf (Sorghum sudanense) were investigated under various concentrations of salinity on their germination (percentage, energy, index and rate), relative of saltinjury rate, length of radicle and hypocotyl. G. soja was germinating adeptly at concentration $<200$ $\mathrm{mmol} / \mathrm{L}, 50 \mathrm{mmol} / \mathrm{L}$ salt level is superior to $S$. sudanense. Seeds of $L$. sinense and $S$. sudanense may germinate at high concentration $400 \mathrm{mmol} / \mathrm{L}$, radicle hypocotyl ratio for last two plants were increment (Li, 2008). Other research, study the effects of various level of $\mathrm{NaCl}(200,400,600$ and $800 \mathrm{mM}$ ) on germination of four plants belong to family Brassicaceae (Sinapis alba and Brasica oleracea) and Solanaceae (Capsicum annum and Solanum lycopersicum) found all the plants only germinated in the lowest level of $\mathrm{NaCl}(200 \mathrm{mM})$ (Bojović et al 2010). Moreover, in ryegrass mean germination time elongated with rising salinity and germination rate decreased. Higher $\mathrm{NaCl}$ concentration lead to a reduction notable of root and shoot length and decreased in there dry and fresh weight also reduce the leaf number (Ilker, 2011).

The enormous genetic variance was found in faba been in terms of seed size and structure in addition to tolerance to several environmental stresses (Duc et al 2010; Hendawey and Younes, 2013). Few reports have been done on seed imbibition and several characteristics of faba been varieties related to stress tolerance. This study was conducted to test the germination under salt stress can distinguish faba been varieties with good salinity tolerance during a crucial germination on five faba bean Egyptian varieties. Therefore, the purpose of the instant study was 1) Appraise the impression of salt stress on various varieties of faba bean 2) Screen out immeasurable salinity resistant faba bean 3) Study the impact of salinity on sprout production of faba bean and to assess the various morphological changes associated with the sprouts under different salinity gradient.

\section{MATERIALS AND METHODS}

Two experiments have been done in Seed Laboratory, Horticulture Department, Faculty of Agriculture, Ain Shams Univ., Cairo, Egypt.

\section{Materials}

Faba been (Vicia faba L.) of five varieties, Nubaria (1), Giza (843), Sakha (1), Sakha (3) and, Sakha (4) were obtained from Agriculture Research Center, Giza, Egypt. $\mathrm{Na} \mathrm{Cl}$ was obtained from El-Gomhoria Company, Cairo, Egypt were used in this experiment.

\section{First experimental design}

The design of the experiment was a factorial complete randomized design with two factors (five faba bean varieties and three salt concentrations) with three replicates for each treatment. The $\mathrm{NaCl}$ 
solutions concentration were $0,1000,2000 \mathrm{ppm}$. 100 Seed of each varieties were soaked in a glass jar method for imbibition up to $18 \mathrm{~h}$. Soaked water was removed and seeds were sprouting for 2 days using method reported by (Abdallah, 2008).

\section{Second experimental design}

The design of the experiment was a factorial complete randomized design with three factors (five faba bean varieties and three salt concentrations as the first experiment) and the third factor is three soaking period ( 0 to $9 \mathrm{~h}, 9$ to $18 \mathrm{~h}$ and 0 to $18 \mathrm{~h}$ ) with three replicates for each treatment.

\section{Water uptake}

Imbibed water was measuring by using three methods. First, was get an initial volume $(400 \mathrm{ml})$ of water in each jar before soaking then after 9hours when changing the soaked solution take up the water only and measuring the residues of soaked water and deduct from initial volume as well as at 18hour similar process. Second water uptake based on weight, seeds in each treatment jar were weighted before soaking (initial weight) and after 18-hour soaking (final imbibition seed weight). Thirdly water uptake based on volume, seeds in each treatment jar were volume measured before soaking (initial volume) and after 9 and18-hour soaking (final imbibition seed volume). For each measurement of seed imbibed water, seeds were accurately removed, drained, quickly dry up by absorbent paper, weighted, and back again into the jar. Imbibed water determined before (Baskin et al 2006; Orozco-Segovia et al 2007; Silva et al 2018) was calculated by following equation:

Water uptake by weight(\%)= $\frac{\text { Final weight-Initial weight }}{\text { Initial weight }} \times 100$

Water uptake by volume(\%)= $\frac{\text { Final volume-Initial volume }}{\text { Initial volume }} \times 100$

\section{Germination Percentage}

Germination percentage was registered for a total of 2 days, and was calculated by the next formulation:

Germination percentage $(\%)=\frac{\text { Number of germinated seeds }}{\text { Number of total seed }} \times 100$

\section{Germination index}

Germination index was calculated as ratio germination percentage of each treatment to germination percentage of control and calculated by the following equation according to (Kandil et al 2012):

Germination Index (\%) = $\frac{\text { Germination \% of each treatment }}{\text { Germination } \% \text { of control }} \times 100$

\section{Salt Tolerance Index (STI)}

Salt tolerance index was calculated as the ratio of the dry plant weight to specific $\mathrm{NaCl}$ concentrations to the dry plant weight of the control and calculated by the next formula according to (Carpıcı et al 2009; Ibrahim et al 2016; Khatun et al 2013):

Salt Tolerance Index (\%) =

$\frac{\text { Total dry weight under NaCl concentration }}{\text { Total dry weightunder control }} \times 100$

\section{Relative salt-injury rate}

Relative salt-injury rate was calculated as subtract between germination percentage of control and germination percentage in $\mathrm{NaCl}$ concentration of each treatment to germination percentage of control and calculated by the next equation ( $\mathbf{L i}$, 2008):

\section{Relative salt-injury rate $=$}

Germination \% of control-Germination \% in $\mathrm{NaCl}$ Germination \%of control

\section{Morphological characteristics}

Seed volume: was measured by water displacement method by following steps; first, fill a beaker with water then registered the baseline initial measurement. Second, submerge seeds inside beaker and registered final measurement. Then, calculated as deduct the initial volume from the final volume (Siswantoro et al 2013) 100 seed.

Sample of 10 sprouts from each replicate were collected for measuring the following character:

Radical length: the radical length measured from the seed to the tip of the radical by centimeters $(\mathrm{cm})$.

Sprout fresh weight: germinated seeds were measured by gram (g). 
Sprout dry weight: germinated seeds recorded by gram (g) after oven drying $60^{\circ} \mathrm{C}$ for 72 hours.

\section{Statistical analysis}

The data were analyzed by analysis of variance (ANOVA) using completely randomized design (Two way analysis of variance for the first experiment and three way analysis for the second experiment). The least significant difference (LSD) at 0.05 levels according to the method described by Snedecor and Cochran, (1980).

\section{RESULTS}

\section{Germination Percentage}

At three $\mathrm{NaCl}$ concentrations, variety Nubaria (1) recorded the highest germination percentage $(75.11 \%)>$ Giza $(843)(73.78 \%)$, whereas Sakha (1) had the lowest percentage (63.56\%). Nubaria (1) and Giza (843) showed higher tolerance to salinity stress more than Sakha genotypes in terms percentage of germination (Table 1). With the increase of salt levels, germination percentage was remarkably decreased. Concerning salinity $x$ variety interaction Nubaria (1) reached the higher germination percentage (80\%) at $\mathrm{NaCl} 1000$ ppm while, Giza (843) had the highest germination percentage of $(68 \%)$ at the $2000 \mathrm{ppm} \mathrm{NaCl}$ concentration (Table 1).

\section{Germination Index \%}

At different salinity levels, Nubaria (1) variety had the higher germination index $(89.42 \%)>$ Giza (843) $(86.53 \%)$, whereas Sakha (1) had the lowest index (79.49\%). Nubaria (1) had higher salt stress tolerance than other varieties using germination index. With the increase concentration of $\mathrm{NaCl}$, germination index was notably decreased. The germination index \% recorded (100\%) in all varieties using distilled water while, Nubaria (1) recorded the higher germination index \%when using $\mathrm{NaCl}$ at $1000 \mathrm{ppm}$ (95.24\%). At the $2000 \mathrm{ppm}$ $\mathrm{NaCl}$ concentration, Giza (843) had the highest germination index of (79.8\%). However, Sakha (1) variety recorded the lowest germination index $(65.84 \%)$ when using saline water at $2000 \mathrm{ppm}$ $\mathrm{NaCl}$ for sprouting (Table 1).

\section{Sprout growth Characteristics}

Data in Table (1) showed also that the differences among varieties $x$ salinity concentrations were significant where increasing salinity concentrations, the length of radical was decreased from $1.71 \mathrm{~cm}$ when using distilled water to $1.14 \mathrm{~cm}$ using 2000 ppm NaCl concentration. Moreover, the variety Sakha (1) recorded the lowest radical length. The interaction showed.

\section{Sprout fresh and dry weight}

Sprout fresh weight was decreased with increasing salinity concentration. While, sprout dry weight did not affected significantly with different salinity concentrations. Nubaria (1) recorded the highest sprout fresh and dry weight while, Giza (843) recorded the lowest sprout fresh and dry weight. Regarding to variety $x$ salinity interaction, data in (Table 1) recorded the highest sprout fresh and dry weight of Nubaria (1) using distilled water while, the lowest sprout fresh and dry weight were recorded using Giza (843) combined with 2000 ppm $\mathrm{NaCl}$ concentration.

\section{Sprout Weight losses \%}

The weight losses indicated significant differences among varieties. Giza (843) had the highest losses (20.66\%) followed by Nubaria (1) (16.59\%) whereas Sakha (1) had the lowest losses of (5.29\%). Salinity concentrations showed significant increase of sprout weight losses $\%$ by increasing the salinity concentrations. The interaction effect showed that sprout weight losses increased with increasing $\mathrm{NaCl}$ concentration, combined with Giza (843) and Nubaria (1) varieties (Table 1).

\section{Imbibed seed weight}

Seed soaking weight for $18 \mathrm{~h}$ increased 100 seed weight from 96.86 to 201.58 (more than twofold). The imbibed seeds weight showed significant differences among varieties, salinity concentrations, soaking time and their interactions (Table 2). Data in (Table 2) recorded the heaviest imbibed seeds weight of Nubaria (1) followed by Sakha (4) \& (3) while the lower weight recorded by Giza (843) and Sakha (1). Using saline water for soaking seeds reduced imbibed seed weight comparing with soaking in distilled water. 
(Vicia faba L) varieties

Table 1. Effect of $\mathrm{NaCl}$ concentration on 2 days old five faba been sprout characters that, the longest radical length was $1.98 \mathrm{~cm}$ recorded by Saka 3 using distilled water while the shortest was $0.65 \mathrm{~cm}$ recorded by Sakha (1) combined with 2000 ppm NaCl concentration (Table 1)

\begin{tabular}{|c|c|c|c|c|c|c|c|}
\hline $\begin{array}{l}\text { Salinity } \\
\text { (NaCl } \\
\text { ppm) }\end{array}$ & Variety & $\begin{array}{c}\text { Germination } \\
\%\end{array}$ & $\begin{array}{l}\text { Germination } \\
\text { Index } \%\end{array}$ & $\begin{array}{l}\text { Radical } \\
\text { length } \\
(\mathrm{cm})\end{array}$ & $\begin{array}{c}\text { Sprout } \\
\text { fresh } \\
\text { weight g } \\
48 \mathrm{~h}\end{array}$ & $\begin{array}{c}\text { Sprout dry } \\
\text { weight g } \\
48 \mathrm{~h}\end{array}$ & $\begin{array}{l}\text { Weight } \\
\text { losses } \%\end{array}$ \\
\hline \multirow[t]{2}{*}{ 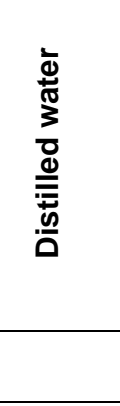 } & $\begin{array}{l}\text { Nubaria (1) } \\
\text { Giza (843) } \\
\text { Sakha (1) } \\
\text { Sakha (3) } \\
\text { Sakha (4) }\end{array}$ & $\begin{array}{l}84 a \\
85.33 a \\
80 a b \\
82.67 a \\
84 a\end{array}$ & $\begin{array}{l}100 a \\
100 a \\
100 a \\
100 a \\
100 a\end{array}$ & $\begin{array}{l}1.81 \mathrm{a} \\
1.74 \mathrm{ab} \\
1.38 \mathrm{bcd} \\
1.98 \mathrm{a} \\
1.65 \mathrm{abc}\end{array}$ & $\begin{array}{l}3.31 \mathrm{a} \\
1.89 \mathrm{fg} \\
2.02 \mathrm{efg} \\
2.3 \mathrm{~cd} \\
2.35 \mathrm{~cd}\end{array}$ & $\begin{array}{l}1.26 \mathrm{a} \\
0.71 \mathrm{~g} \\
0.81 \mathrm{fg} \\
0.88 \mathrm{~cd} \\
0.91 \mathrm{c}\end{array}$ & $\begin{array}{l}7.35 \mathrm{j} \\
19.27 \mathrm{e} \\
0.89 \mathrm{j} \\
7.88 \mathrm{i} \\
9.83 \mathrm{~h} \\
\end{array}$ \\
\hline & Mean & $83.2 \mathrm{~A}$ & $100 \mathrm{~A}$ & $1.71 \mathrm{~A}$ & $2.37 \mathrm{~A}$ & $0.92 \mathrm{~A}$ & $9.04 \mathrm{~B}$ \\
\hline \multirow[t]{2}{*}{$\begin{array}{l}\text { 틈 } \\
\text { 응 }\end{array}$} & $\begin{array}{l}\text { Nubaria (1) } \\
\text { Giza (843) } \\
\text { Sakha (1) } \\
\text { Sakha (3) } \\
\text { Sakha (4) }\end{array}$ & $\begin{array}{l}80 \mathrm{ab} \\
68 \text { abcde } \\
65.33 \mathrm{cde} \\
77.33 \mathrm{abc} \\
74.67 \mathrm{abcd}\end{array}$ & $\begin{array}{l}95.24 \mathrm{a} \\
79.8 \mathrm{bc} \\
81.64 \mathrm{bc} \\
93.56 \mathrm{ab} \\
89.2 \mathrm{ab}\end{array}$ & $\begin{array}{l}1.68 \mathrm{abc} \\
1.64 \mathrm{abc} \\
0.95 \text { ef } \\
1.59 \mathrm{abc} \\
1.18 \mathrm{de}\end{array}$ & $\begin{array}{l}2.83 \mathrm{~g} \\
1.84 \mathrm{~g} \\
2.05 \mathrm{dfg} \\
2.24 \mathrm{cde} \\
2.29 \mathrm{~cd} \\
\end{array}$ & $\begin{array}{l}1.08 \mathrm{~b} \\
0.7 \mathrm{~g} \\
0.78 \mathrm{efg} \\
0.86 \mathrm{cde} \\
0.91 \mathrm{c}\end{array}$ & $\begin{array}{l}20.79 \mathrm{c} \\
20 \mathrm{~d} \\
5.34 \mathrm{I} \\
10.81 \mathrm{~g} \\
9.76 \mathrm{~h} \\
\end{array}$ \\
\hline & Mean & 73.07 B & 87.89 B & $1.41 \mathrm{~B}$ & $2.25 \mathrm{~B}$ & $0.87 \mathrm{~A}$ & 13.34 A \\
\hline \multirow{6}{*}{$\begin{array}{l}\text { E } \\
\text { 응 } \\
\text { ᄋ }\end{array}$} & Nubaria (1) & $61.33 \mathrm{de}$ & $73.01 \mathrm{~cd}$ & $1.3 \mathrm{cde}$ & $2.78 \mathrm{~b}$ & $1.07 \mathrm{~b}$ & $21.63 \mathrm{~b}$ \\
\hline & Giza (843) & 68 bcde & $79.8 \mathrm{bc}$ & $1.67 \mathrm{abc}$ & $1.81 \mathrm{~g}$ & $0.68 \mathrm{~g}$ & $22.7 \mathrm{a}$ \\
\hline & Sakha (1) & $45.33 f$ & $56.84 \mathrm{e}$ & $0.65 f$ & $2.02 \mathrm{efg}$ & 0.74 def & $9.65 \mathrm{~m}$ \\
\hline & Sakha (3) & 54.67 ef & $65.74 \mathrm{de}$ & $1.14 \mathrm{de}$ & $2.39 \mathrm{c}$ & $0.9 \mathrm{~cd}$ & $5.82 \mathrm{k}$ \\
\hline & Sakha (4) & $58.67 \mathrm{e}$ & 69.42 cde & 0.91 ef & 2.15 cdef & 0.86 cde & $15.23 \mathrm{f}$ \\
\hline & Mean & $57.6 \mathrm{C}$ & $68.96 \mathrm{C}$ & $1.14 \mathrm{C}$ & $2.23 \mathrm{~B}$ & $0.85 \mathrm{~A}$ & $15 \mathrm{~A}$ \\
\hline \multirow{5}{*}{ 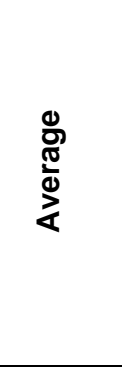 } & Nubaria (1) & $75.11 \mathrm{~A}$ & $89.42 \mathrm{~A}$ & $1.6 \mathrm{~A}$ & $2.97 \mathrm{~A}$ & $1.13 \mathrm{~A}$ & $16.59 \mathrm{~A}$ \\
\hline & Giza (843) & $73.78 \mathrm{~A}$ & $86.53 \mathrm{AB}$ & $1.69 \mathrm{~A}$ & $1.85 \mathrm{D}$ & $0.7 \mathrm{D}$ & $20.66 \mathrm{~A}$ \\
\hline & Sakha (1) & $63.56 \mathrm{~B}$ & $79.49 \mathrm{~B}$ & $0.99 \mathrm{C}$ & $2.03 \mathrm{C}$ & $0.78 \mathrm{C}$ & $5.29 \mathrm{C}$ \\
\hline & Sakha (3) & $71.56 \mathrm{~A}$ & $86.43 \mathrm{AB}$ & $1.57 \mathrm{~A}$ & $2.31 \mathrm{~B}$ & $0.88 \mathrm{~B}$ & $8.17 \mathrm{~B}$ \\
\hline & Sakha (4) & $72.44 \mathrm{~A}$ & $86.21 \mathrm{AB}$ & $1.25 \mathrm{~B}$ & $2.26 \mathrm{~B}$ & $0.9 \mathrm{~B}$ & $11.6 \mathrm{~B}$ \\
\hline
\end{tabular}


Regarding the varieties and salinity interaction, data showed that the highest imbibed seeds weight recorded by Nubaria (1) combined with distilled water whereas, the lowest imbibed seeds weight was recorded by Giza (843) with the highest salinity concentration (2000 ppm NaCl) (Table 2).

\section{Water Uptake\%}

Water uptake based on weight $(\mathrm{g} / 100$ seed) or on volume ( $\mathrm{ml} / 100$ seed) was decreased by increasing the salinity concentration. While, varieties showed no significant differences regarding water uptake $\%$ based on weight ( $g / 100$ seed) while Sakha (4) recorded the highest water uptake based on volume ( $\mathrm{ml} / 100$ seed). Regarding the interaction between varieties and salinity, data recorded the highest water uptake by weight recorded by Giza (843) combined with 1000 ppm $\mathrm{NaCl}$ concentration. The lowest water uptake by weight was recorded by Sakha (1) using 2000 ppm $\mathrm{NaCl}$ concentration. Also, water uptake\% based on 100 seed volume interaction between varieties and salt concentrations at $18 \mathrm{~h}$, Sakha (4) recorded the highest water uptake\% (140.43\%) using distilled water while, in Sakha (3) using 1000 ppm NaCl concentration recorded the lowest water uptake by weight (98.33\%) (Table 2).

\section{Relative salt injury rate}

Data of salt relative injury rate presented in Table (2) Data illustrated significant differences among varieties, salinity and their interaction. The salt injury rate increased by increasing of $\mathrm{NaCl}$ concentration. Nubaria (1) had better tolerance to salt stress than Sakha (1) variety in terms of salt injury. The Relative salt injury rate recorded no damage in all varieties using distilled water while, Sakha varieties recorded the highest injury when using $2000 \mathrm{ppm} \mathrm{NaCl}$ concentration data was more pronounced with Sakha (1) (Table 2).

\section{Salt Tolerance Index (STI).}

Concerning salt tolerance index at germination stage, data indicated significant differences among varieties. Sakha (3) recorded the highest salt tolerance index while Sakha (1) recorded the lowest. On the other hand, data showed no significant differences regarding salinity concentrations. The index $\%$ in soaking $x$ variety recorded $(100 \%)$ in all varieties using distilled water while, Sakha (4) pre- sented better salt stress tolerance than other varieties at $1000 \mathrm{ppm} \mathrm{NaCl}$ and Sakha (3) at 2000 ppm $\mathrm{NaCl}$ concentration (Table 2).

\section{Seed imbibed water}

Water imbibition during soaking period was significantly affected by variety, salinity and soaking period and their interactions (Table 3). Nubaria (1) recorded the highest water imbibition amount of water $(114.07 \mathrm{ml})$ while, the lowest water imbibition was recorded by Sakha (1). Water absorption of seeds by decreased by increasing $\mathrm{NaCl}$ concentration. Concerning soaking period, data showed that the highest water imbibition recorded within 18hour soaking, moreover the absorption of water in the first 9-hour soaking $(92.53 \mathrm{ml})$ was doubled than the absorption in the second 9-hour soaking (46.68 $\mathrm{ml})$. Concerning varieties and soaking period interaction, data showed that the highest water imbibition recorded by Nubaria (1) $(171.1 \mathrm{ml})$ within 18 hours soaking whereas, the lowest water imbibition was recorded by Sakha (1) (122.14 ml) and Giza (843) (123.45 ml). However, Nubaria (1) recorded about $61.6 \%$ of water absorption within the beginning 9 hour and the other $38.4 \%$ was absorbed in the last 9 hours of soaking. On the other hand, the lowest water imbibition recorded by Sakha (1) variety $(74.2 \%$ of water absorption within the first 9 hour of soaking) and the rest $25.8 \%$ of absorbed water was recorded in the second 9 hour of soaking. Concerning varieties, salinity and soaking period interaction, data in (Table 3 ) showed that the highest water imbibition recorded by Nubaria (1) (189.91 $\mathrm{ml}$ ) in total 18 hour soaking and the lowest water imbibition recorded by Sakha (1) (28 $\mathrm{ml}$ )within second 9 hour soaking using distilled water for soaking.

\section{Seed volume $\left(\mathrm{cm}^{3}\right)$}

The seed volume $\left(\mathrm{cm}^{3}\right)$ of 100 seeds decreased by increasing salinity concentration. The seed volume decreased from $\left(145.67 \mathrm{~cm}^{3}\right)$ to $\left(132.26 \mathrm{~cm}^{3}\right)$. Regarding the effect of varieties, data showed that there were significant differences among verities concerning seed volume $\left(\mathrm{cm}^{3}\right)$ of 100 seeds. Nubaria (1) recorded the highest volume $\left(181.73 \mathrm{~cm}^{3}\right)$ while, the lowest volume recorded $\left(112.97 \mathrm{~cm}^{3}\right.$ ) by Giza (843). Regarding soaking period, data showed that the seed volume $\left(\mathrm{cm}^{3}\right)$ of 100 seeds increased by soaking seeds for 9 hour by about $81.2 \%$ while soaking for 18 hours increased seed volume by about $19.6 \%$ than the volume of seeds soaked for 9 hours. 
(Vicia faba L) varieties

Table 2. Effect of 100 seed soaking for $18 \mathrm{~h}$ in saline water on weight, water uptake\%, salt injury and tolerance of five faba bean varieties

\begin{tabular}{|c|c|c|c|c|c|c|c|c|}
\hline \multirow[b]{2}{*}{$\begin{array}{c}\text { Salinity } \\
(\mathrm{NaCl} \\
\mathrm{ppm})\end{array}$} & \multicolumn{4}{|c|}{100 seed Weight $(\mathrm{g})$} & \multirow[b]{2}{*}{\begin{tabular}{|c|}
$\begin{array}{c}\text { Water } \\
\text { uptake\% by } \\
\text { weight }(g / 100 \\
\text { seed) }\end{array}$ \\
\end{tabular}} & \multirow[b]{2}{*}{$\begin{array}{c}\text { Water } \\
\text { uptake } \% \text { by } \\
\text { volume }(\mathrm{ml} / \\
100 \text { seed) } \\
\end{array}$} & \multirow[b]{2}{*}{$\begin{array}{c}\text { Relative } \\
\text { salt injury } \\
\text { rate }\end{array}$} & \multirow[b]{2}{*}{$\begin{array}{c}\text { Salt } \\
\text { tolerance } \\
\text { index \% }\end{array}$} \\
\hline & Variety & $\begin{array}{l}\text { Zero time } \\
\text { (row seed) }\end{array}$ & $\begin{array}{c}\text { Total } 18 \text { hour } \\
\text { (imbibed } \\
\text { seed) }\end{array}$ & Mean & & & & \\
\hline 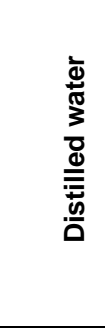 & $\begin{array}{l}\text { Nubaria (1) } \\
\text { Giza (843) } \\
\text { Sakha (1) } \\
\text { Sakha (3) } \\
\text { Sakha (4) }\end{array}$ & $\begin{array}{l}134.93 \mathrm{~g} \\
80.27 \mathrm{hi} \\
82.93 \mathrm{hi} \\
96.8 \mathrm{hi} \\
100.67 \mathrm{~h}\end{array}$ & $\begin{array}{l}290.96 \text { a } \\
167.63 \text { ef } \\
178.13 \text { ef } \\
202.77 \text { c } \\
206.13 \text { c }\end{array}$ & $\begin{array}{l}212.95 \mathrm{~A} \\
123.95 \mathrm{DE} \\
130.53 \mathrm{DE} \\
149.79 \mathrm{C} \\
153.4 \mathrm{C} \\
\end{array}$ & $\begin{array}{l}115.67 \mathrm{a} \\
109.17 \mathrm{abc} \\
114.86 \mathrm{ab} \\
109.64 \mathrm{abc} \\
104.72 \mathrm{abc}\end{array}$ & $\begin{array}{l}119.84 \text { abcde } \\
102.8 \mathrm{de} \\
133.36 \text { abcde } \\
125.82 \mathrm{abcd} \\
140.43 \mathrm{a}\end{array}$ & $\begin{array}{l}0.0 \mathrm{f} \\
0.0 \mathrm{f} \\
0.0 \mathrm{f}\end{array}$ & $\begin{array}{l}100 a b \\
100 a b \\
100 a b \\
100 a b \\
100 a b\end{array}$ \\
\hline \multirow[b]{2}{*}{$\begin{array}{l}\varepsilon \\
\frac{0}{2} \\
8 \\
ㅇ\end{array}$} & Mean & $99.12 \mathrm{C}$ & $209.13 \mathrm{~A}$ & 154.12 A & $110.81 \mathrm{~A}$ & 124.45 A & $0.0 \mathrm{C}$ & $100 \mathrm{~A}$ \\
\hline & $\begin{array}{l}\text { Nubaria (1) } \\
\text { Giza (843) } \\
\text { Sakha (1) } \\
\text { Sakha (3) } \\
\text { Sakha (4) }\end{array}$ & $\begin{array}{l}122.13 \mathrm{~g} \\
76.8 \mathrm{i} \\
84.53 \mathrm{hi} \\
95.47 \mathrm{hi} \\
100.27 \mathrm{~h}\end{array}$ & $\begin{array}{l}249.94 \text { b } \\
166.12 \text { ef } \\
181.63 \text { de } \\
202.82 \text { c } \\
204.31 \mathrm{c} \\
\end{array}$ & $\begin{array}{l}186.04 \mathrm{~B} \\
121.46 \mathrm{DE} \\
133.08 \mathrm{DE} \\
149.15 \mathrm{C} \\
152.29 \mathrm{C} \\
\end{array}$ & $\begin{array}{l}104.74 \mathrm{abc} \\
116.21 \mathrm{a} \\
114.86 \mathrm{ab} \\
112.44 \mathrm{abc} \\
103.93 \mathrm{abc}\end{array}$ & $\begin{array}{l}102.23 \text { abcde } \\
117.86 \text { abcde } \\
131.46 \mathrm{ab} \\
98.33 \mathrm{e} \\
126.67 \mathrm{abc}\end{array}$ & $\begin{array}{l}0.05 \text { ef } \\
0.2 \mathrm{cde} \\
0.18 \mathrm{~cd} \\
0.07 \mathrm{def} \\
0.11 \mathrm{def}\end{array}$ & $\begin{array}{l}85.09 \mathrm{c} \\
99.73 \mathrm{ab} \\
96.30 \mathrm{ab} \\
96.95 \mathrm{ab} \\
100.26 \mathrm{ab}\end{array}$ \\
\hline \multirow{3}{*}{$\begin{array}{l}\text { E } \\
\text { 응 } \\
\text { 유 }\end{array}$} & Mean & $95.84 \mathrm{C}$ & $200.97 \mathrm{~B}$ & $148.4 \mathrm{~B}$ & $110.44 \mathrm{~A}$ & $116.04 \mathrm{~B}$ & $0.12 \mathrm{~B}$ & $97.42 \mathrm{~A}$ \\
\hline & $\begin{array}{l}\text { Nubaria (1) } \\
\text { Giza (843) } \\
\text { Sakha (1) } \\
\text { Sakha (3) } \\
\text { Sakha (4) }\end{array}$ & $\begin{array}{l}120.8 \mathrm{~g} \\
77.6 \mathrm{i} \\
85.33 \mathrm{hi} \\
96 \mathrm{hi} \\
98.4 \mathrm{~h}\end{array}$ & $\begin{array}{l}246.5 \mathrm{~b} \\
158.65 \mathrm{f} \\
170.81 \mathrm{ef} \\
199.67 \mathrm{~cd} \\
197.57 \mathrm{~cd}\end{array}$ & $\begin{array}{l}183.65 \mathrm{~B} \\
118.13 \mathrm{E} \\
128.07 \mathrm{DE} \\
147.84 \mathrm{C} \\
147.98 \mathrm{C}\end{array}$ & $\begin{array}{l}104.24 \mathrm{abc} \\
104.27 \mathrm{abc} \\
99.78 \mathrm{c} \\
107.97 \mathrm{abc} \\
100.61 \mathrm{bc}\end{array}$ & $\begin{array}{l}116.79 \text { bcde } \\
107.16 \text { cde } \\
102.02 \mathrm{e} \\
106.83 \mathrm{cde} \\
127.82 \mathrm{abc}\end{array}$ & $\begin{array}{l}0.27 \mathrm{bc} \\
0.2 \mathrm{~cd} \\
0.43 \mathrm{a} \\
0.34 \mathrm{ab} \\
0.31 \mathrm{abc}\end{array}$ & $\begin{array}{l}98.98 \mathrm{ab} \\
96.65 \mathrm{ab} \\
91.36 \mathrm{bc} \\
105.87 \mathrm{a} \\
93.99 \mathrm{bc}\end{array}$ \\
\hline & Mean & $95.63 \mathrm{C}$ & 194.64 B & $145.13 \mathrm{~B}$ & 103.37 B & $112.13 \mathrm{~B}$ & $0.31 \mathrm{~A}$ & $100.08 \mathrm{~A}$ \\
\hline \multirow[t]{2}{*}{$\begin{array}{l}\frac{9}{\pi} \\
\frac{\pi}{\Phi} \\
\frac{\pi}{8}\end{array}$} & $\begin{array}{l}\text { Nubaria (1) } \\
\text { Giza (843) } \\
\text { Sakha (1) } \\
\text { Sakha (3) } \\
\text { Sakha (4) }\end{array}$ & $\begin{array}{l}125.96 \mathrm{E} \\
78.22 \mathrm{G} \\
84.27 \mathrm{G} \\
96.09 \mathrm{~F} \\
99.78 \mathrm{~F}\end{array}$ & $\begin{array}{l}262.47 \mathrm{~A} \\
164.14 \mathrm{D} \\
176.86 \mathrm{C} \\
201.76 \mathrm{~B} \\
202.67 \mathrm{~B}\end{array}$ & $\begin{array}{l}194.21 \mathrm{~A} \\
121.18 \mathrm{D} \\
130.56 \mathrm{C} \\
148.92 \mathrm{~B} \\
151.22 \mathrm{~B}\end{array}$ & $\begin{array}{l}108.22 \mathrm{~A} \\
109.88 \mathrm{~A} \\
109.83 \mathrm{~A} \\
110.02 \mathrm{~A} \\
103.09 \mathrm{~A}\end{array}$ & $\begin{array}{l}112.95 \mathrm{BC} \\
109.28 \mathrm{C} \\
122.28 \mathrm{AB} \\
110.33 \mathrm{BC} \\
131.64 \mathrm{~A}\end{array}$ & $\begin{array}{l}0.11 \mathrm{~B} \\
0.13 \mathrm{AB} \\
0.2 \mathrm{~A} \\
0.14 \mathrm{AB}\end{array}$ & $\begin{array}{l}94.69 \mathrm{~B} \\
98.79 \mathrm{AB} \\
93.83 \mathrm{C} \\
100.94 \mathrm{~A} \\
98.08 \mathrm{AB}\end{array}$ \\
\hline & time & $96.86 \mathrm{~B}$ & $201.58 \mathrm{~A}$ & & & & & \\
\hline
\end{tabular}


Table 3. Effect of soaking period in saline water on water imbibition ( $\mathrm{ml} / 100 \mathrm{seed})$ and seed volume $\left(\mathrm{cm}^{3}\right.$ $/ 100$ seed) of five faba bean varieties

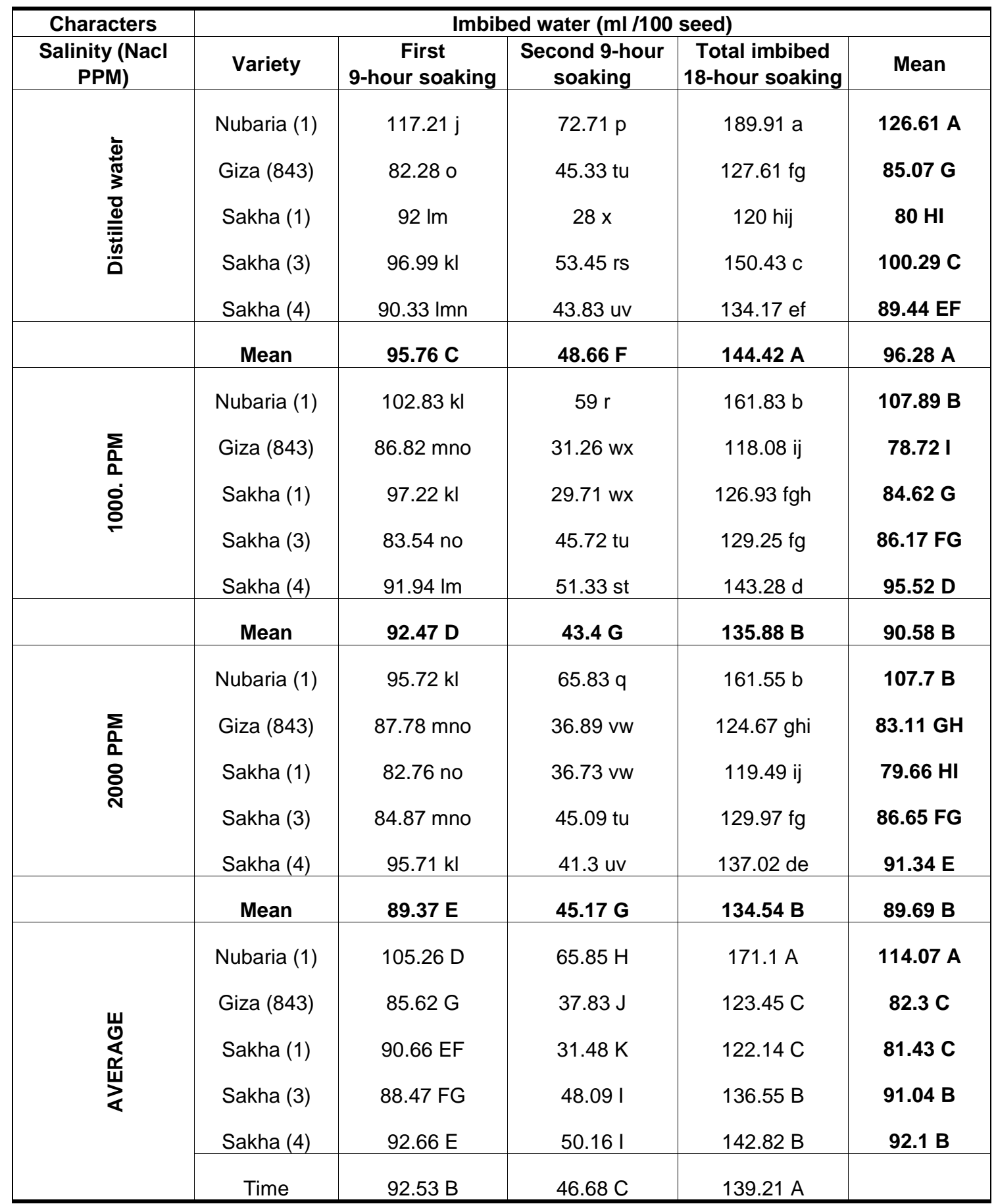


Table 3. Cont.

\begin{tabular}{|c|c|c|c|c|c|}
\hline \multirow{2}{*}{$\begin{array}{c}\text { Characters } \\
\text { Salinity } \\
\text { (Nacl ppm) }\end{array}$} & \multicolumn{5}{|c|}{ Seed volume $\left(\mathrm{cm}^{3} / 100\right.$ seed $)$} \\
\hline & Variety & $\begin{array}{c}\text { Non soaked } \\
\text { (row seed) }\end{array}$ & $\begin{array}{l}\text { First 9-hour } \\
\text { soaking }\end{array}$ & $\begin{array}{l}\text { Total 8-hour } \\
\text { soaking }\end{array}$ & Mean \\
\hline \multirow[t]{2}{*}{ 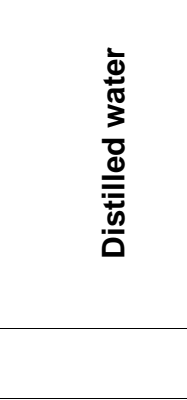 } & $\begin{array}{l}\text { Nubaria (1) } \\
\text { Giza (843) } \\
\text { Sakha (1) } \\
\text { Sakha (3) } \\
\text { Sakha (4) }\end{array}$ & $\begin{array}{l}122.67 \text { mnop } \\
72 r \\
69.33 r \\
86.67 \mathrm{qr} \\
80 \mathrm{r}\end{array}$ & $\begin{array}{l}212.58 \mathrm{bc} \\
121.33 \text { nop } \\
153.33 \mathrm{ghijkl} \\
143.83 \mathrm{ijklmn} \\
158.17 \mathrm{ghijkl}\end{array}$ & $\begin{array}{l}269.97 \mathrm{a} \\
145.89 \mathrm{ijklmn} \\
161.33 \mathrm{fghijk} \\
195.71 \mathrm{~cd} \\
192.22 \mathrm{cde}\end{array}$ & $\begin{array}{l}201.74 \mathrm{~A} \\
113.07 \mathrm{GH} \\
128 \mathrm{DEF} \\
142.07 \mathrm{CD} \\
143.46 \mathrm{C}\end{array}$ \\
\hline & Mean & 86.13 E & $157.85 \mathrm{C}$ & 193.03 A & 145.67 A \\
\hline \multirow[t]{2}{*}{$\begin{array}{l}\sum \\
\frac{2}{0} \\
8 \\
\delta\end{array}$} & $\begin{array}{l}\text { Nubaria (1) } \\
\text { Giza (843) } \\
\text { Sakha (1) } \\
\text { Sakha (3) } \\
\text { Sakha (4) }\end{array}$ & $\begin{array}{l}116 p \\
68 r \\
72 r \\
85.33 q r \\
81.33 r\end{array}$ & $\begin{array}{l}172.62 \text { defgh } \\
134.39 \text { Imno } \\
156.36 \text { ghijkl } \\
160.54 \text { fghijkl } \\
157 \text { ghijkl }\end{array}$ & $\begin{array}{l}232.12 \text { b } \\
147.98 \text { hijklm } \\
166.38 \text { fghij } \\
169.17 \text { efghi } \\
184.56 \text { def }\end{array}$ & $\begin{array}{l}173.58 \mathrm{~B} \\
116.9 \mathrm{FGH} \\
131.58 \mathrm{CDE} \\
138.35 \mathrm{CD} \\
140.96 \mathrm{CD}\end{array}$ \\
\hline & Mean & $84.53 \mathrm{E}$ & $156.18 \mathrm{C}$ & 180.04 B & 140.27 B \\
\hline \multirow[t]{2}{*}{$\begin{array}{l}\sum_{0} \\
\frac{0}{2} \\
\text { 을 }\end{array}$} & $\begin{array}{l}\text { Nubaria (1) } \\
\text { Giza (843) } \\
\text { Sakha (1) } \\
\text { Sakha (3) } \\
\text { Sakha (4) }\end{array}$ & $\begin{array}{l}108 p q \\
65.33 r \\
76 r \\
80 r \\
77.33 r\end{array}$ & $\begin{array}{l}168.28 \text { efghij } \\
121.78 \text { nop } \\
136.02 \text { klmno } \\
142.24 \text { jklmn } \\
144.17 \text { ijklmn }\end{array}$ & $\begin{array}{l}233.33 \text { b } \\
135.39 \text { klmno } \\
154.43 \text { ghijkl } \\
164.98 \text { fghij } \\
176.6 \text { defg }\end{array}$ & $\begin{array}{l}196.87 \mathrm{~B} \\
107.5 \mathrm{H} \\
122.15 \mathrm{EFG} \\
129.08 \mathrm{CDEF} \\
132.07 \mathrm{CDE}\end{array}$ \\
\hline & Mean & 81.33 E & 142.5 D & 172.95 B & 132.026 B \\
\hline \multirow[t]{2}{*}{ 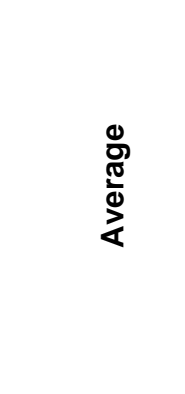 } & $\begin{array}{l}\text { Nubaria (1) } \\
\text { Giza (843) } \\
\text { Sakha (1) } \\
\text { Sakha (3) } \\
\text { Sakha (4) }\end{array}$ & $\begin{array}{l}115.56 \mathrm{E} \\
68.44 \mathrm{G} \\
72.44 \mathrm{FG} \\
84 \mathrm{~F} \\
79.56 \mathrm{FG}\end{array}$ & $\begin{array}{l}184.49 \mathrm{~B} \\
125.94 \mathrm{E} \\
148.57 \mathrm{CD} \\
148.87 \mathrm{CD} \\
153.11 \mathrm{CD}\end{array}$ & $\begin{array}{l}245.14 \mathrm{~A} \\
143.09 \mathrm{D} \\
160.72 \mathrm{C} \\
176.62 \mathrm{~B} \\
184.46 \mathrm{~B}\end{array}$ & $\begin{array}{l}181.73 \mathrm{~A} \\
112.47 \mathrm{D} \\
127.24 \mathrm{C} \\
136.5 \mathrm{~B} \\
139.04 \mathrm{~B}\end{array}$ \\
\hline & Time & $84 \mathrm{C}$ & 152.2 B & $182.01 \mathrm{~A}$ & \\
\hline
\end{tabular}


Regarding to varieties, soaking time interaction, data showed the highest seed volume recorded by Nubaria (1) $\left(245.14 \mathrm{~cm}^{3}\right)$ for 18 hour soaking, whereas, the lowest seed volume were recorded in all variety row seeds and Giza (843) $\left(125.94 \mathrm{~cm}^{3}\right)$ in the first 9 hour soaking only. The interaction between varieties and salinity presented that Nubaria (1) had the highest seed volume (201.74 $\mathrm{cm}^{3}$ ) using distilled water. On the contrary, data showed that Giza (843) recorded lowest seed volume $\left(107.5 \mathrm{~cm}^{3}\right)$ using higher $\mathrm{NaCl}$ concentration (2000 ppm). The interaction between variety, soaking period and salinity recorded, the higher seed volume of Nubaria (1) $\left(269.97 \mathrm{~cm}^{3}\right)$ within 18 hour soaking, whereas, the lowest seed volume was recorded in row seed varieties and Giza (843) in the 1 st 9 hour soaking in both distilled water and saline water (2000 ppm NaCl) within the $2 \mathrm{nd} 9 \mathrm{hr}$ of soaking in distilled water (Table 3 ).

\section{DISCUSSION}

Quality of germination and good sprouting are important for plant emergence and development. Good germination, enough to produce seedlings for plant population and edible sprouts. The seed germination quality is expressed by characters such as percentage and index of germination. Seedlings healthy growth showed good radical growth, which increased best population of plant, particularly in saline conditions. Therefore, a test of germination could be useful tool using it for selection and development varieties for production salted soil or production of edible sprouts using saline water especially in arid zone (Abdallah et al 1991; Ibrahim et al 2016; Khan and Gulzar, 2003; Zhou et al 2010).

Five varieties of faba bean from Egypt showed similar trends to salinity stress with wheat Ibrahim et al (2016); Kandil et al (2012) but in our faba bean experiment, their differences in reduction of water uptake, percentage of germination, and radical initiation. The differences suggest that a test of germination may help in suitable faba bean varieties selected for saline zone soils or for sprout production in arid zone.

In this study, a decreasing in water uptake by weight or volume was showed with $\mathrm{NaCl}$ concentrations increasing in faba bean varieties. These agreement with wheat and sorghum reports by Ibrahim et al (2016); Kader and Jutzi, (2002). The osmotic barrier owing to $\mathrm{NaCl}$ concentration affected water uptake (Mehmet, 2006). Faba bean data similar to other researchers (Akbarimoghaddam et al 2011; Ilker, 2011; Mehmet, 2006), who reported that germination of seed and earlier growth of seedling decreased in saline soils and water with different varieties responses. The germination of seed affected by $\mathrm{NaCl}$ may be related to generate an external osmotic potential which restrict water of uptake. Moreover, several studies demonstrated reducing of water absorption in ryegrass, wheat and barley (Bağci et al 2003; Ibrahim et al 2016; Ilker, 2011) under abiotic stress such as salinity or water stresses.

In the current study, the lower percentage of germination and germination index was recorded in $2000 \mathrm{ppm} \mathrm{NaCl}$ concentration. The decline in percentage and index of germination using $\mathrm{NaCl}$ solution attributed to combined effect of osmotic pressure as reported by Moud and Maghsoudi, (2008); Munns, (2002) and salt concentrations toxicity (Ibrahim et al 2016; Mehmet, 2006; Munns and Tester, 2008) or owing to effect of $\mathrm{Cl}^{-}$ (Almodares et al 2007) which increase osmotic stress. Higher $\mathrm{Na}^{+}$and $\mathrm{Cl}^{-}$ions uptake in germination using saline solution can induce toxicity for cells which slows or inhibits the proportion of germination and finally reduces percentage of germination (Taiz and Zeiger, 2003). In our research, the varieties responses differently to three different $\mathrm{NaCl}$ concentrations. Data means that there are genetic variations regarding to varieties in respect of abiotic saline stress tolerance. Increasing $\mathrm{NaCl}$ concentration showed decrease in percentage and index of germination in all faba bean varieties. Some of the varieties were more tolerant than the others. Result of salinity with variety interaction showed that the germination percentage of varieties at $\mathrm{NaCl} 1000$ ppm Nubaria (1) reached the highest germination percentage (80\%) while, Giza (843) had the highest germination percentage of (68\%) at the $2000 \mathrm{ppm} \mathrm{NaCl}$ concentration. Similar results were recorded by $\mathbf{L i}, \mathbf{( 2 0 0 8 )}$ in Kuntze, Sieb and Stapf; Zhang et al (2010) in barely Kumari and Vishnuvardhan, (2015) in Kodo millet; El-Bastawisy et al (2018) in faba bean. Begum et al (2010) indicated that salt stress induced delay and decrease in germination percentage through higher accumulation of $\mathrm{Na}^{+}$and $\mathrm{Cl}^{-}$ and lower water uptake.

The radical length is powerful character for salinity stress since radical is direct touch the media and uptake water from the media or saline solutions and supply it to the whole plant. For this sense, radical length provides a great evidence for 
plant response to salt stress (Al-Karaki, 1997; Carpıcı et al 2009; Ibrahim et al 2016). Reduction in growth as a result of saline stress also published by other researcher in other species $\mathbf{L i},(2008)$ in Kuntze, Sieb and Stapf; Carpıcı et al., (2009) om maize; Ibrahim et al 2016 on wheat. The increase in salinity concentration decreased the length of radical and dry weight of faba bean varieties. Similar data reported by Hussein et al (2007); Carpıcı et al (2009) in maize. Genotypes responded variously to salt stress.

Faba bean sprout fresh and dry weight were also recorded in the varieties which having higher germination percentage. Nubaria (1) recorded the highest sprout fresh \& dry weight while, Giza (843) recorded the lowest fresh \& dry sprout weight. The results in this study agree with Hussein et al (2007) in maize and Ibrahim et al (2016) in wheat. They recorded negative relationship identified between vegetative growth characters and increasing salinity. In addition, the sprout dry weight losses \% indicated significant differences among varieties. Giza (843) had the highest losses (20.66\%) followed by Nubaria (1) (16.59\%) whereas Sakha (1) had the lowest losses of (5.29\%). Salinity concentrations showed significant differences regarding sprout weight losses\%. This is agree with the results of other researchers (Bahrami and Razmjoo, 2012; Begum et al 2013; Ibrahim et al 2016).

Salt tolerance of faba bean varieties decreased as the $\mathrm{NaCl}$ concentrations increased. The lowest salt tolerance index was registered in 2000 ppm $\mathrm{NaCl}$ concentration. These results agreed with Carpıcı et al (2009) in maize; Kumari and Vishnuvardhan, (2015) in spinach Alom et al (2016) and Ibrahim et al (2016) in wheat; Kan et al (2016) in soybean.

As the $\mathrm{NaCl}$ concentration increases the relative salt injury rate was increased in all faba bean varieties. The relative salt injury rate recorded no damage in all varieties using distilled water while, Sakha varieties recorded the highest injury when using 2000 ppm NaCl concentration data was more pronounced with Sakha (1). This damage increment may be due to change in cell expansion process which is controlled by processes related to cellular water uptake and cell wall extension (Ibrahim et al 2019; Kumari and Vishnuvardhan, 2015; Li, 2008).

Water imbibition was significantly affected by variety, salinity and soaking period and their interactions. Nubaria (1) recorded the highest imbibed amount of water $(114.07 \mathrm{ml})$ while, the lowest imbibed water (81.43 $\mathrm{ml}$ ) was recorded in Sakha (1). The seeds water absorption in saline solutions decreased with of $\mathrm{NaCl}$ concentration increase. Concerning soaking period data showed the higher imbibed water within 18 hour soaking, moreover the absorption of water in the first 9-hour soaking $(92.53 \mathrm{ml})$ was almost twice the absorption in the second 9-hour soaking $(46.68 \mathrm{ml})$ similar data was obtained by Akbarimoghaddam et al (2011); Ibrahim et al (2016); Ilker, (2011); Kader and Jutzi, (2002); Mehmet, (2006). Begum et al (2010) reported the accumulation of sodium and Chloride ions increased with increasing salinity. The result indicated that salinity induced lower water absorption (Begum et al 2010).

The volume $\left(\mathrm{cm}^{3}\right)$ of 100 seeds in $\mathrm{NaCl}$ concentration decreased with increasing concentration, seed volume was decreased from (145.67 $\mathrm{cm}^{3}$ ) to $\left(132.26 \mathrm{~cm}^{3}\right)$. Volume of seed $\mathrm{s}$ showed a highly significant difference among varieties, Nubaria (1) recorded the highest volume (181.73 $\mathrm{cm}^{3}$ ) while, the lowest volume was recorded (112.97 $\mathrm{cm}^{3}$ ) in Giza (843). Regarding soaking period data showed that the imbibed water for 9 hour increased row seed volume by about $81.2 \%$ while soaking for 18 hours increased volume by about $116.7 \%$ than row seeds and about $19.6 \%$ than the volume of seeds soaked for 9 hours. Panuccio et al (2014) also found the volume decrease with increasing salinity through lower water absorption.

\section{CONCLUSION}

It can be concluded the uptake of water decreased with increasing salinity. Giza (843) and Sakha (3) varieties had recorded the highest tolerance to salinity. These genotypes were more tolerant to salinity and recommended to use them in breeding program for increasing faba bean production in Egypt. Therefore, these genotypes could be grown on slightly saline condition. However, this could not be a guarantee for these faba bean genotype to be salt-tolerant in later stages of plant growth. There must be additional experimental research to be conducted at the mature plant growth stage and then recommended salt concentration that provide best separation of faba bean varieties on the stage of germination and vegetative stage. 


\section{REFERENCES}

Abdallah M.M. 2008. Seed Sprouts, a Pharaoh's Heritage to Improve Food Quality. Arab Universities J. of Agric. Sci. 16, 469-478.

Abdallah M.M., Jones R., El-Beltagy A. and Elmore C. 1991. Interaction of Salinity and Herbicides in Tomato. Yemen J. Agric. Sci. 1, 108-121.

Akbarimoghaddam H., Galavi M., Ghanbari A. and Panjehkeh N. 2011. Salinity Effects on Seed Germination and Seedling Growth of Bread Wheat Cultivars. Trakia J. of Sci. 9, 4350.

Al-Karaki G.N. 1997. Barley responce to salt stress at varied levels of phosphorous., J. of Plant Nutrition (USA). 20, 1635-1643.

Almodares A. and Hadi D. 2007. Effectd of salt stress on germination prsentge and seedleing growth in sweet sorghum cult. Biological Sci. 7, 1492-1495.

Alom R., Hasan M.A., Islam M.R. and Wang Q.F. 2016. Germination characters and early seedling growth of wheat (Triticum aestivum L.) genotypes under salt stress conditions. J. of Crop Sci. and Biotechnology. 19, 383-392.

Bağci S.A., Ekiz H. and Yilmaz A. 2003. Determination of the salt tolerance of some barley genotypes and the characteristics affecting tolerance. Turkish J. of Agric. and Forestry 27, 253-260.

Bahrami H. and Razmjoo J. 2012. Effect of salinity stress $(\mathrm{NaCl})$ on germination and early seedling growth of ten sesame cultivars (Sesamum indicum L.). Int. J. of Agric. Sci. 2, 529-537.

Baskin J.M., Baskin C.C. and Dixon K.W. 2006. Physical dormancy in the endemic Australian genus Stylobasium, a first report for the family Surianaceae (Fabales). Seed Sci. Research 16, 229-232.

Begum F., Ahmed I., Nessa A. and Sultana W. 2010. The effect of salinity on seed quality of wheat. J. of the Bangladesh Agric. Univ. 8, 19-22.

Begum M.A.J., Selvaraju P. and Venudevan B. 2013. Saline stress on seed germination. Acedemic J. 8, 1420-1423.

Bojović B., Đelić G., Topuzović M. and Stanković M. 2010. Effects of $\mathrm{NaCl}$ on seed germination in some species from families Brassicaceae and Solanaceae. Kragujevac J. Sci. 32, 83-87.

Carpıcı E.B., Celık N. and Bayram G. 2009. Effects of salt stress on germination of some maize (Zea mays L.) cultivars. African J. of Biotechnology 8, 4918-4922.
Copeland L.O., Lawrence O. and McDonald M.B. 2001. Principles of seed science and technology. Kluwer Academic Publishers.

Duc G., Bao S., Baum M., Redden B., Sadiki M., Suso M.J., Vishniakova M. and Zong X. 2010. Diversity maintenance and use of Vicia faba $\mathrm{L}$. genetic resources. Field Crops Research. 115, 270-278.

El-Bastawisy Z.M., El-Katony T.M. and Abd ElFatah S.N. 2018. Genotypic variability in salt tolerance of Vicia faba during germination and early seedling growth. J. of King Saud Univ., Sci. 30, 270-277.

Erba D., Angelino D., Marti A., Manini F., Faoro F., Morreale F., Pellegrini N. and Casiraghi M.C. 2019. Effect of sprouting on nutritional quality of pulses. Int. J. of Food Sci. and Nutrition 70, 30-40.

FAO 2017. FAOSTAT. URL http://www.fao.org/faostat/en/

Ghazi A.K. 1998. Seed size and water potential effects on water uptake, germination and growth of lentil. J. of Agronomy and Crop Sci. 181, 237-242.

Hendawey M.H. and Younes A.M.A. 2013. Biochemical evaluation of some faba bean cultivars under rainfed conditions at El-Sheikh Zuwayid. Annals of Agric. Sci. 58, 183-193.

Hossain M.S. and Mortuza M.G. 2006. Chemical Composition of Kalimatar, a Locally Grown Strain of Faba Bean (Vicia faba L.). Pakistan J. of Biological Sci. 9, 1817-1822.

Hussein M.M., Gaballah M.S. and Balbaa L.K. 2007. Salicylic Acid and Salinity Effects on Growth of Maize Plants. Research J. of Agric. and Biological Sci. 3, 321-328.

Ibrahim M.E.H., Zhu X., Zhou G. and Nimir N.E.A. 2016. Comparison of germination and seedling characteristics of wheat varieties from China and Sudan under salt stress. Agronomy J. 108, 85-92.

Ibrahim M.H., Abas N.A. and Zahra S.M. 2019. Impact of Salinity Stress on Germination of Water Spinach (Ipomoea aquatica). Annual Research \& Review in Biology. pp. 1-12.

Ilker N. 2011. Effects of salinity stress on water uptake, germination and early seedling growth of perennial ryegrass. African J. of Biotechnology 10, 10418-10424.

Jamil M., Deog Bae L., Kwang Yong J., Ashraf M., Sheong Chun L. and Eui Shik R. 2006. Effect of Salt ( $\mathrm{Nacl})$ Stress on Germination and Early Seedling Growth of Four Vegetables Species. J. of Central European Agric. 7, 273282. 
(Vicia faba L) varieties

Kader M.A. and Jutzi S.C. 2002. Temperature, osmotic pressure and seed treatments influence imbibition rates in sorghum seeds. $J$. of Agronomy and Crop Sci., 188, 286-290.

Kan G., Ning L., Li Y., Hu Z., Zhang W., He X. and Yu D. 2016. Identification of novel loci for salt stress at the seed germination stage in soybean. Breeding Sci., 66, 530-541.

Kandil A., Sharief A. and Elokda M. 2012. Germination and Seedling Characters of Different Wheat Cultivars under Salinity Stress. J. of Basic and Applied Sci., 8, 585-596.

Khan M.A. and Gulzar S. 2003. Germination responses of Sporobolus ioclados: a saline desert grass. J. of Arid Environments 53, 387394.

Khan M.A., Ungar I.A. and Showalter A.M. 2000. Effects of sodium chloride treatments on growth and ion accumulation of the halophyte Haloxylon recurvum. Communications in Soil Sci. and Plant Analysis. 31, 2763-2774.

Khatun M., Hafiz M.H.R., Hakim M. and Siddiqui M.N. 2013. Responses of Wheat Genotypes to Salt Stress in Relation to Germination and Seedling Growth. Int. J. of Bio-resource and Stress Management 4, 635-640.

Kumari R.P. and Vishnuvardhan Z. 2015. Effect of salinity on growth, protein and antioxidant enzymes in three Kodo millet (Paspalum scrobiculatum) germplasm. Int. J. of Current Microbiology and Applied Sci., 4, 475-483.

Kumawat S., Gothwal D.K., Kumawat K.R. and Sharma R.K.M. 2017. Effect of salt stress on seed germination and early seedling traits in fenugreek (Trigonella foenum-graecum L.) genotypes grown under different salinity levels, J. of Pharmacognosy and Phytochemistry 6(5), 776-781.

Li Y. 2008. Effect of salt stress on seed germination and seedling growth of three salinity plants. Pakistan J. of Biological Sci., PJBS. 11, 1268-1272.

Manz, B., 2005. Water Uptake and Distribution in Germinating Tobacco Seeds Investigated in Vivo by Nuclear Magnetic Resonance Imaging. Plant Physiology 138, 1538-1551.

Mehmet A., 2006. Effects of $\mathrm{NaCl}$ on the Germination, Seedling Growth and Water Uptake of Triticale. Turkish J. of Agric. and Forestry 30, 39-47.

Moud A.M. and Maghsoudi K. 2008. Salt Stress Effects on Respiration and Growth of Germinated Seeds of Different Wheat (Triticum aestivum L.) Cultivars. World J. of Agric. Sci. 4, 351-358.
Munns, R., 2002. Comparative physiology of salt and water stress. Plant, cell \& environment. 25, 239-250.

Munns R. and Tester M. 2008. Mechanisms of Salinity Tolerance. Annual Review of Plant Biology 59, 651-681.

Okçu G., Kaya M.D. and Atak M. 2005. Effects of salt and drought stresses on germination and seedling growth of pea (Pisum sativum L.). Turkish J. of Agric. and Forestry., 29, 237242.

Orozco-Segovia A., Márquez-Guzmán J., Sánchez-Coronado M.E., Gamboa De Buen A., Baskin J.M. and Baskin C.C. 2007. Seed anatomy and water uptake in relation to seed dormancy in Opuntia tomentosa (Cactaceae, Opuntioideae). Annals of Botany. 99, 581-592.

Panuccio M.R., Jacobsen S.E., Akhtar S.S. and Muscolo A. 2014. Effect of saline water on seed germination and early seedling growth of the halophyte quinoa. AoB PLANTS. 6, plu 047.

Santos J.M.A.P. dos, Francisco de A. de Oliveira, J.F. de M., Targino, Ana J. de O., Santos, Costa, L.P. da and Dos S.T. 2018. Saline stress and potassium/calcium ratio in fertigated eggplant. Revista Brasileira de Engenharia Agrícola e Ambiental., 22, 770775.

Schopfer P. and Plachy C. 1984. Control of Seed Germination by Abscisic Acid: II. Effect on Embryo Water Uptake in Brassica napus $\mathrm{L}$. Plant Physiology 76, 155-160.

Silva B.M. da S.E., Silva C. de O.E., Môro F.V. and Vieira R.D. 2018. Seed anatomy and water uptake and their relation to seed dormancy of Ormosia paraensis Ducke. J. of Seed Sci., 40, 237-245.

Siswantoro J., Prabuwono A.S. and Abdulah A. 2013. Volume Measurement of Food Product with Irregular Shape Using Computer Vision and Monte Carlo Method: A Framework. Procedia Technology 11, 764-770.

Snedecor G.W. and Cochran W.G. 1980. Statistical Methods. 7th Edition, lowa State University Press, Ames.

Taiz, L. and Zeiger E. 2003. Plant physiology. 3rd edn., Annals of Botany. Oxford Univ. Press.

Zhang H., Irving L.J., McGill C., Matthew C., Zhou D. and Kemp P. 2010. The effects of salinity and osmotic stress on barley germination rate: sodium as an osmotic regulator. Annals of Botany 106, 1027-1035.

Zhou G., Ma B.L, Li J., Feng C., Lu J. and Qin, P. 2010. Determining Salinity Threshold Level for Castor Bean Emergence and Stand Establishment. Crop Sci. 50, 2030-2036. 


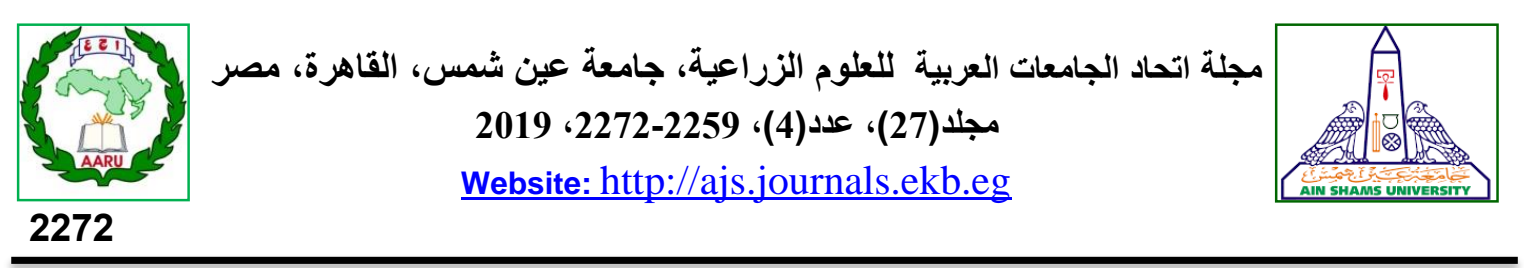

تأثير الملوحة على صفات النبت لخمسة أصناف من الفول

[180]

محمود عادل أحمد على" - ممدوح محمد فوزي عبدالله - نشوي عطية أبو العزم - أحمد أبو اليزيد

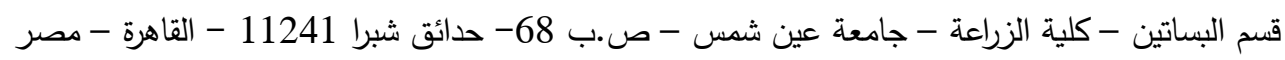

*Corresponding author: mahmoud adel489@agr.asu.edu.eg

Received 25 July, 2019 Accepted 12 November, 2019

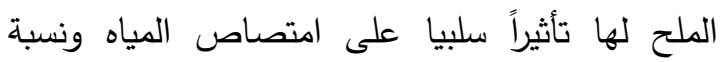
الإنبات وطول الجذير، الوزن الطازج والجاف للنبت، الإنياه

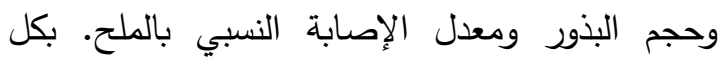

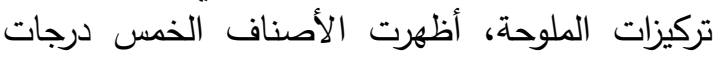

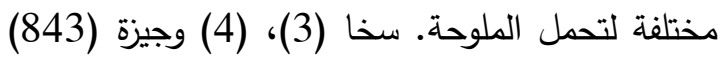
أفضل مؤشر لتحمل الملوحة من نوبارية (1) (1) وجيزة (1) وسخا

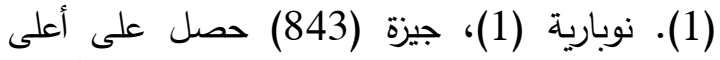
نسبة إنبات ومؤشر الإنبات، وسخا (1) (1) كان أقصر القرل طول الجذير وأكثر حساسية لمعدل الإنيات وسذا لإندابة بالملح.

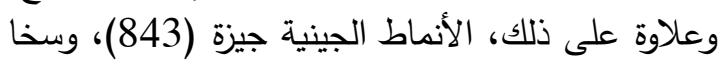

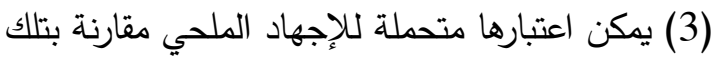

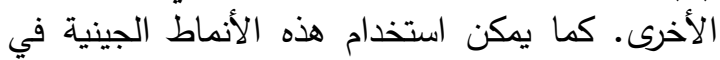

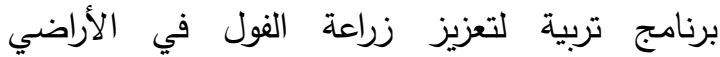

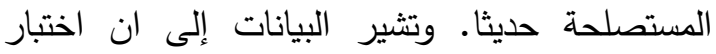

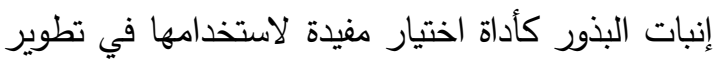
سلالات فول جديدة للإنتاج في التربة المالحة.

الكلمات الدالة: إنبات بذور فول الفول، امتصاص

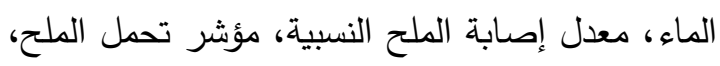
شخصية البرعم، إجهاد الملوحة الماتة

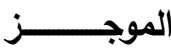

تتمثل إحدى الخصائص الفريدة في المراحل الاولي الإني من النبات هو الانبات. الإنبات هو التعبير الظاهري

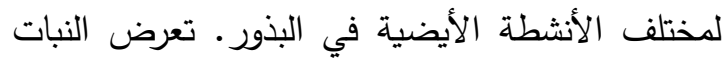

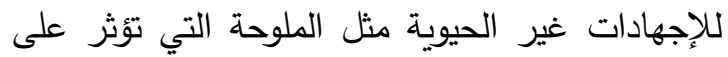

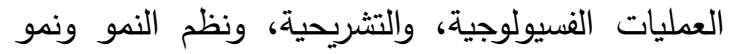

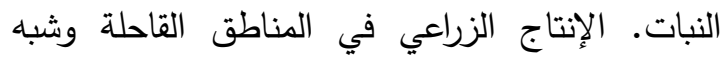
القاحلة منخفض يعزي للعديد من العوامل على سبيل الإنتاجليل المثال تراكم الأملاح في التربة والمياه، مجموع مساحة لإند الأراضي تحت الملوحة هو حوالي 953 مليون هكتار . الهدف من هذا البحث كان لتقييم صفات التئ الإنبات لخمسة أصناف من الفول [نوبارية (1)، جئية (1)، هذات (843)، سخا (1)، سخا (3)، سخا (4)] تم استخدام المحلول الملحي الذي يحتوي على (10، 1000 و 2000 جزء في المليون كلوريد الصوديوم). واستخدمت لدراسة

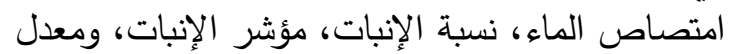

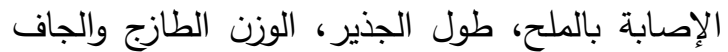

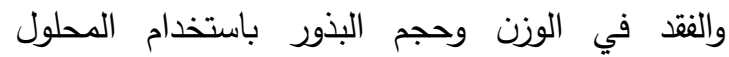
الملحي. وأثارت النتائج إلى أن التركيزات العالية من 
